\title{
Serum YKL-40 as predictor of outcome in hypersensitivity pneumonitis
}

\author{
Xiaoping Long ${ }^{1,2}$, Xuan $\mathrm{He}^{1}$, Shinichiro Ohshimo ${ }^{3}$, Matthias Griese ${ }^{4}$, \\ Rafael Sarria ${ }^{5}$, Josune Guzman ${ }^{6}$, Ulrich Costabel $^{1}$ and Francesco Bonella ${ }^{1}$ \\ Affiliations: ${ }^{1}$ Interstitial and Rare Lung Disease Unit, Ruhrlandklinik, University of Duisburg-Essen, Essen, \\ Germany. ${ }^{2}$ Dept of Respiratory Medicine, The First Affiliated Hospital of University of South China, Hengyang, \\ P.R. China. ${ }^{3}$ Dept of Molecular and Internal Medicine, Graduate School of Biomedical Sciences, Hiroshima \\ University, Hiroshima, Japan. ${ }^{4} \mathrm{Dr}$. von Haunersches Kinderspital, University of Munich, Member of the \\ German Center for Lung Research, Munich, Germany. ${ }^{5}$ Dept of Neurosciences, Faculty of Medicine and \\ Dentistry, Basque Country University, Bilbao, Spain. ${ }^{6}$ General and Experimental Pathology, Ruhr University, \\ Bochum, Germany.
}

Correspondence: Francesco Bonella, Interstitial and Rare Lung Disease Unit, Ruhrlandklinik, University of Duisburg-Essen, 45239 Essen, Germany. E-mail: francesco.bonelladruhrlandklinik.uk-essen.de

@ERSpublications

YKL-40 could help clinicians to assess disease activity and outcome of patients with hypersensitivity pneumonitis http://ow.ly/U4Qw304PTJ5

Cite this article as: Long $\mathrm{X}$, He X, Ohshimo S, et al. Serum YKL-40 as predictor of outcome in hypersensitivity pneumonitis. Eur Respir J 2017; 49: 1501924 [https://oi.org/10.1183/13993003.01924-2015].

ABSTRACT YKL-40, a chitinase-like protein mainly secreted by macrophages, neutrophils and epithelial cells, is increased in patients with idiopathic interstitial pneumonia and sarcoidosis. We aimed to investigate the role of YKL-40 as a biomarker in hypersensitivity pneumonitis (HP).

$72 \mathrm{HP}$ patients, 100 interstitial lung disease (ILD) controls and 60 healthy controls were studied. YKL40 was measured by ELISA in serum and bronchoalveolar lavage fluid (BALF) at baseline and follow-up. The relationship between YKL-40 levels, clinical variables and disease outcome was evaluated.

Baseline serum YKL-40 levels were significantly higher in HP patients than in healthy controls $(\mathrm{p}<0.001)$, but lower than in patients with other ILDs. Baseline BALF YKL-40 levels in HP patients were the highest among ILD patients. In HP patients, serum YKL-40 correlated with the diffusing capacity of the lung for carbon monoxide at baseline $(\mathrm{p}<0.01)$ and over time $(\mathrm{p}<0.001)$. HP patients whose disease progressed or who died had higher baseline YKL-40 levels than those who remained stable and survived $(\mathrm{p}<0.001)$. At a cut-off of $119 \mathrm{ng} \cdot \mathrm{mL}^{-1}$, the baseline serum YKL-40 level predicted disease progression (hazard ratio 6.567; $\mathrm{p}<0.001$ ), and at a cut-off of $150 \mathrm{ng} \cdot \mathrm{mL}^{-1}$ was associated with mortality (hazard ratio 9.989; $\mathrm{p}<0.001)$.

Serum YKL-40 may be a useful prognostic biomarker in HP patients.

Support statement: This study was supported by Arbeitsgemeinschaft zur Förderung der Pneumologie an der Ruhrlandklinik (AFPR). Funding information for this article has been deposited with the Open Funder Registry.

Conflict of interest: None declared.

Copyright OERS 2017 


\section{Introduction}

Hypersensitivity pneumonitis (HP), also called extrinsic allergic alveolitis, is a T-cell-driven immunologic lung disease caused by repeated exposure to organic particles in susceptible subjects [1,2]. Type III and type IV hypersensitivity reactions are involved in the pathogenesis of HP [3]. HP may progress to severe pulmonary fibrosis $[1,4]$. Therefore, there is a need to identify noninvasive biomarkers that can predict disease outcome in HP patients.

YKL-40 is a chitinase-like protein [5] mainly secreted by macrophages, neutrophils and epithelial cells, which is involved in the inflammatory response to tissue injury [6, 7]. It is best known as a biomarker for diseases that are characterised by inflammation, fibrosis and tissue remodelling [6, 8-10]. For instance, circulating levels of YKL-40 have been found to be associated with liver fibrosis [9], Crohn's disease [11] and rheumatoid arthritis [12]. Recently, YKL-40 has been proposed as a diagnostic and prognostic biomarker for various forms of interstitial lung disease (ILD), especially idiopathic pulmonary fibrosis (IPF) and sarcoidosis $[8,13,14]$. Whether YKL-40 is also associated with HP is unknown.

The aim of the present study was to determine the clinical utility of YKL-40 as a biomarker in HP patients. We hypothesised that YKL-40 is elevated in HP patients and that YKL-40 level is associated with disease outcome. Some of the results of this study have been previously reported in an abstract [15].

\section{Materials and methods \\ Study subjects}

We retrospectively studied $72 \mathrm{HP}$ patients and $100 \mathrm{ILD}$ controls (45 patients with IPF, 34 with idiopathic nonspecific interstitial pneumonia (iNSIP) and 21 with cryptogenic organising pneumonitis (COP)), who were followed in our institution between January 2007 and December 2013. 60 volunteers served as healthy controls. The diagnosis of HP and ILD was based on clinical/high-resolution computed tomography (HRCT) findings, bronchoalveolar lavage fluid (BALF) characteristics and/or histopathological findings on biopsy [1, 16-19]. Acute, subacute and chronic HP were defined as previously described [1, 16]. The study was approved by the local institutional review board (approval number 15-6486-BO) and the experiments complied with current laws in Germany. Informed consent was obtained from the ILD patients and healthy controls.

\section{Definition of disease progression in HP}

Disease progression was defined as deterioration of self-reported symptoms (worsening of dyspnoea, cough, chest pain and weight loss), and/or lung function (decrease in forced vital capacity (FVC) $>10 \%$ and/or diffusing capacity of the lung for carbon monoxide (DLCO) $\geqslant 15 \%)$, and/or chest imaging (increase in existing or appearance of new infiltrates compatible with HP) since the last follow-up visit [20, 21]. Otherwise, the patients were defined as stable/improved.

\section{YKL-40 and lactate dehydrogenase laboratory assays}

Serum samples were obtained from all patients at the first evaluation and in $39 \mathrm{HP}$ patients also during follow-up at intervals ranging from 6 to 36 months. A total of 59 follow-up measurements were obtained. The samples were stored at $-20^{\circ} \mathrm{C}$ or $-80^{\circ} \mathrm{C}$ until analysis. Bronchoalveolar lavage was performed via a fibreoptic bronchoscope as previously described [22]; the supernatant was collected and stored at $-80^{\circ} \mathrm{C}$. YKL-40 in serum and BALF was measured by ELISA (Quidel, San Diego, CA, USA) as previously described [23]. Lactate dehydrogenase $(\mathrm{LDH})$ in serum was routinely measured (normal value for $\mathrm{LDH}<225 \mathrm{U} \cdot \mathrm{L}^{-1}$ ).

\section{Pulmonary function tests and blood gas analysis}

Measurements including FVC, forced expiratory volume in $1 \mathrm{~s}$ (FEV1), total lung capacity (TLC), DLCO, arterial oxygen tension $\left(\mathrm{PaO}_{2}\right)$, arterial carbon dioxide tension $\left(\mathrm{PaCO}_{2}\right)$, arterial oxygen saturation $\left(\mathrm{SaO}_{2}\right)$ and alveolar-arterial oxygen tension difference $\left(\mathrm{PA}_{\mathrm{A}-\mathrm{O}} \mathrm{O}_{2}\right)$ were performed at the time of blood sample collection. Values were expressed as percentages of predicted normal values [24].

\section{Statistical analysis}

Continuous variables were evaluated for a normal distribution with the Kolmogorov-Smirnov test. Parametric data are presented as mean \pm SEM. Categorical variables are presented as either a percentage of the total or numerically, as appropriate. Multiple comparisons were performed by one-way ANOVA and least significant difference or Dunnett's post hoc tests. Comparison between two groups was done with t-test or Wilcoxon's rank test for continuous variables, and Chi-squared or Fisher's exact test for categorical variables. Spearman's correlation coefficient was obtained for correlations. Receiver operating characteristic (ROC) analysis was used to test the role of baseline YKL-40 in serum and BALF as a diagnostic marker of HP and a predictor of disease outcome. Univariate and multivariate Cox's proportional hazard regression models were used to analyse prognostic factors. The Kaplan-Meier method with log-rank test was used to analyse 
whether baseline YKL-40 levels were associated with disease outcome. p-values of $<0.05$ were considered statistically significant. All statistical analyses were performed using SPSS 17.0 (SPSS Inc., Chicago, IL, USA).

\section{Results}

\section{Characteristics of study subjects}

Demographic and laboratory characteristics of the enrolled subjects are shown in table 1 . The mean \pm SEM follow-up time of the HP patients was $38 \pm 2$ months (range 1-60) from initial blood collection. Of the 72 HP patients, 11 presented with the acute/subacute form and 61 presented with the chronic form of HP. Relevant antigen exposures were fungi/moulds (4\%), birds/feathers (74\%), both (21\%) or unknown (1\%). During follow-up, $29 \mathrm{HP}$ patients experienced disease progression (symptom worsening in 100\%, lung function deterioration in $90 \%$, chest imaging worsening in 38\%). No patient had disease progression on the basis of symptoms alone. $16 \mathrm{HP}$ patients died. The majority of patients in all groups were not treated with corticosteroids at baseline; these were those patients who were enrolled at the time of diagnosis, before treatment was started.

\section{Baseline serum and BALF levels of $Y K L-40$}

Serum YKL-40 levels were significantly higher in HP patients $\left(127 \pm 9 \mathrm{ng} \cdot \mathrm{mL}^{-1}\right)$ than in healthy controls $\left(39 \pm 4 \mathrm{ng} \cdot \mathrm{mL}^{-1} ; \mathrm{p}<0.001\right)$, but lower than in IPF $\left(214 \pm 20 \mathrm{ng} \cdot \mathrm{mL}^{-1} ; \mathrm{p}<0.001\right)$, iNSIP $\left(184 \pm 21 \mathrm{ng} \cdot \mathrm{mL}^{-1}\right.$; $\mathrm{p}<0.05)$ and COP $\left(213 \pm 33 \mathrm{ng} \cdot \mathrm{mL}^{-1} ; \mathrm{p}<0.05\right)$ patients (figure 1a). Among all ILD patients, serum YKL-40 levels were higher in males than females $\left(213 \pm 14\right.$ versus $\left.163 \pm 12 \mathrm{ng} \cdot \mathrm{mL}^{-1} ; \mathrm{p}<0.01\right)$ and in smokers than nonsmokers $\left(214 \pm 13\right.$ versus $\left.161 \pm 12 \mathrm{ng} \cdot \mathrm{mL}^{-1} ; \mathrm{p}<0.01\right)$. Serum YKL-40 levels were higher in untreated patients than in those treated with corticosteroids ( $206 \pm 11$ versus $\left.141 \pm 13 \mathrm{ng} \cdot \mathrm{mL}^{-1} ; \mathrm{p}<0.001\right)$. Among HP patients, serum YKL-40 levels were higher in patients with acute/subacute disease than in those with chronic disease $\left(179 \pm 27\right.$ versus $117 \pm 9 \mathrm{ng} \cdot \mathrm{mL}^{-1}$; $\left.\mathrm{p}<0.05\right)$.

\section{TABLE 1 Demographics and characteristics of all enrolled subjects}

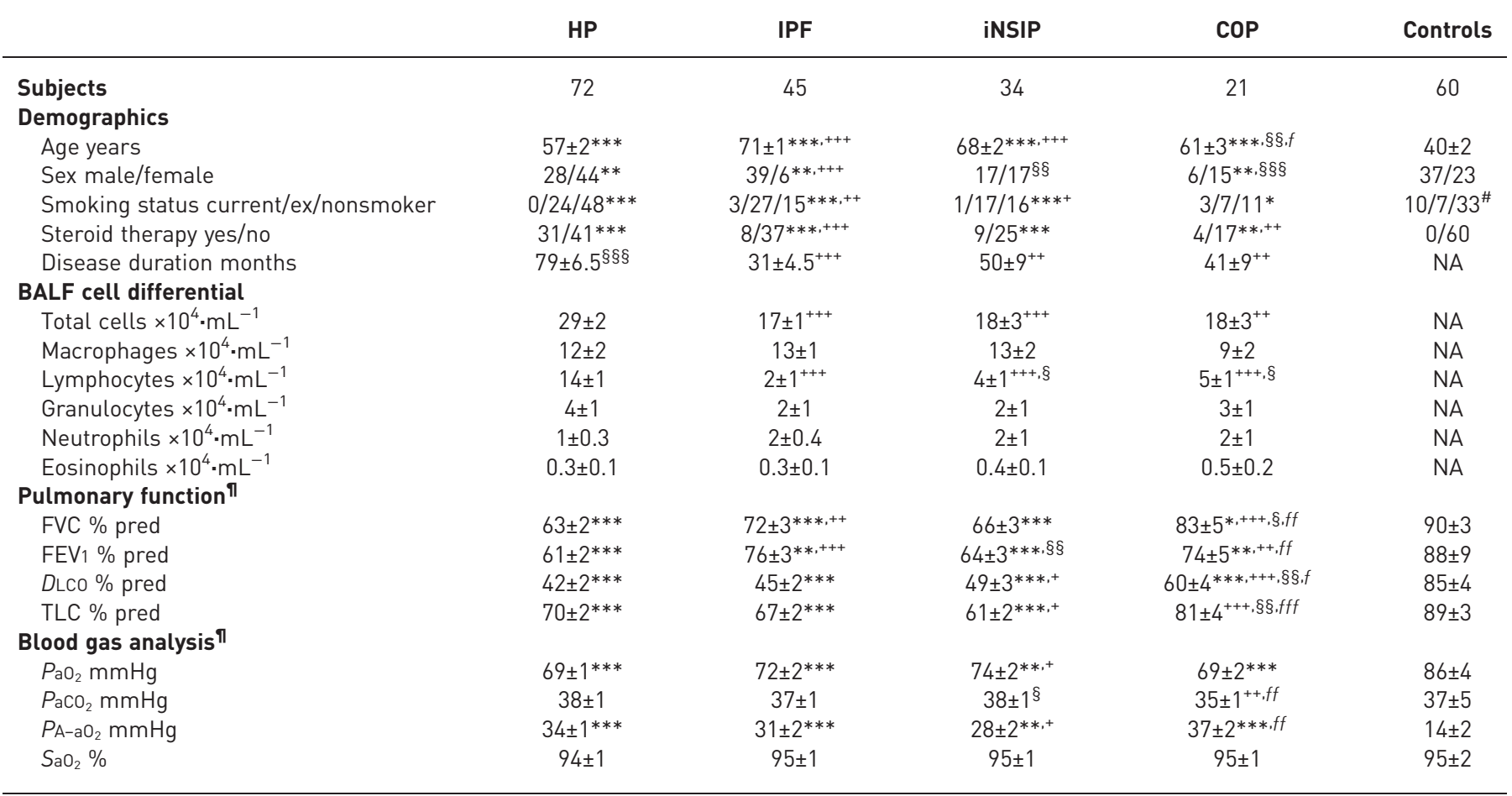

Data are presented as mean \pm SEM unless otherwise stated. HP: hypersensitivity pneumonitis; IPF: idiopathic pulmonary fibrosis; iNSIP: idiopathic nonspecific interstitial pneumonia; COP: cryptogenic organising pneumonitis; NA: not available; BALF: bronchoalveolar lavage fluid; FVC: forced vital capacity; FEV1: forced expiratory volume in $1 \mathrm{~s} ; D \mathrm{LCO}$ : diffusing capacity of the lung for carbon monoxide; TLC: total lung capacity; $\mathrm{PaO}_{2}$ : arterial oxygen tension; $\mathrm{PaCO}_{2}$ : arterial carbon dioxide tension; $P_{\mathrm{A}-\mathrm{aO}}$ : alveolar-arterial oxygen tension difference; $\mathrm{SaO}_{2}$ : arterial oxygen saturation. \#: smoking status was available in 50 healthy controls; ": pulmonary function and blood gas analysis were available in 18 healthy controls. ${ }^{*}: p<0.05,{ }^{* *}: p<0.01,{ }^{* * *}: p<0.001$, versus controls. ${ }^{+}: p<0.05,{ }^{++}: p<0.01,{ }^{+++}: p<0.001$, versus HP. ${ }^{\S}: p<0.05, \S \S$ : $p<0.01, \S \S \S$, $p<0.001$, versus IPF. ${ }^{f}: p<0.05,{ }^{f f}: p<0.01,{ }^{f f f}: p<0.001$, versus iNSIP. 
a)

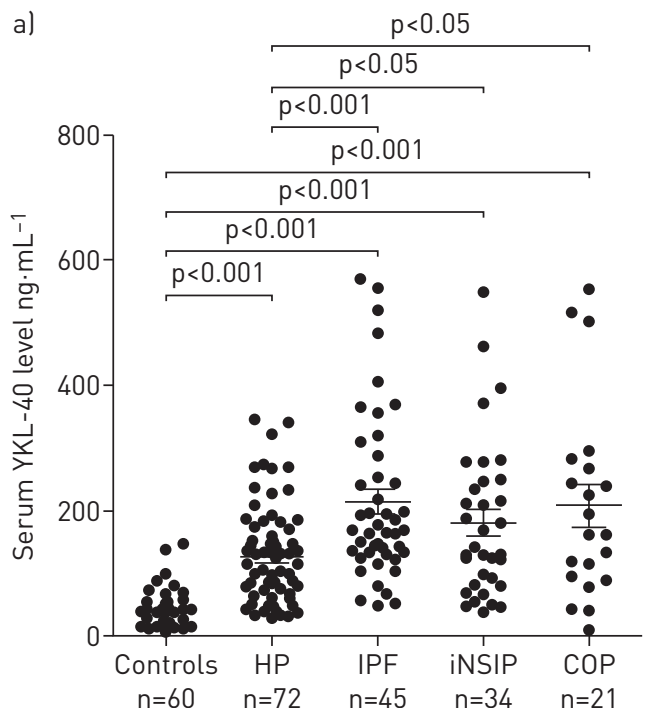

b)

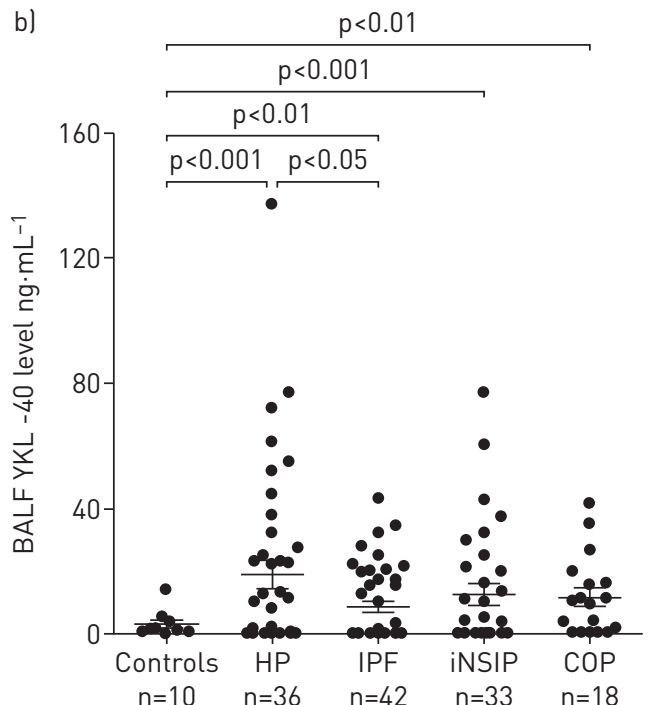

FIGURE 1 a) Serum and b) bronchoalveolar lavage fluid (BALF) YKL-40 levels in the studied subjects at baseline. Dots represent single patients. Bars represent mean \pm SEM values. COP: cryptogenic organising pneumonitis; HP: hypersensitivity pneumonitis; iNSIP: idiopathic nonspecific interstitial pneumonia; IPF: idiopathic pulmonary fibrosis.

BALF YKL-40 levels were significantly higher in HP patients $\left(21 \pm 5 \mathrm{ng} \cdot \mathrm{mL}^{-1}\right)$ than in healthy controls ( $\left.3 \pm 1 \mathrm{ng} \cdot \mathrm{mL}^{-1} ; \mathrm{p}<0.001\right)$ and in those with IPF $\left(9 \pm 2 \mathrm{ng} \cdot \mathrm{mL}^{-1} ; \mathrm{p}<0.05\right)$ (figure $\left.1 \mathrm{~b}\right)$. BALF YKL-40 levels were higher in patients with acute/subacute HP patients than in patients with chronic HP (42 \pm 10 versus $\left.15 \pm 7 \mathrm{ng} \cdot \mathrm{mL}^{-1} ; \mathrm{p}<0.05\right)$. No effect of sex, smoking or treatment on BALF levels was observed (data not shown).

Using ROC analysis, at a cut-off of $>47 \mathrm{ng} \cdot \mathrm{mL}^{-1}$, serum YKL-40 levels showed the best sensitivity (88\%), specificity (77\%) and accuracy (88\%) to discriminate HP from healthy controls (area under the curve (AUC) $0.904 ; \mathrm{p}<0.001$ ), and at a cut-off of $>134 \mathrm{ng} \cdot \mathrm{mL}^{-1}$, serum YKL-40 levels showed the best sensitivity (76\%), specificity $(61 \%)$ and accuracy $(71 \%)$ to discriminate HP from IPF (AUC 0.727; $p<0.001$ ). For BALF YKL-40 levels, no significantly predictive cut-off value was found.

Correlations between serum YKL-40 and other characteristics in HP

Baseline serum YKL-40 levels positively correlated with baseline serum LDH levels $(\mathrm{r}=0.554, \mathrm{p}<0.001)$, BALF total cell counts $(\mathrm{r}=0.496, \mathrm{p}<0.001)$ and BALF lymphocyte counts $(\mathrm{r}=0.451, \mathrm{p}<0.001)$; a weak correlation was seen with age $(\mathrm{r}=0.264, \mathrm{p}<0.05)$. Serum YKL-40 levels inversely correlated with DLCO $(\mathrm{r}=-0.310, \mathrm{p}<0.01)$ (figure $2 \mathrm{a})$ and $\operatorname{FVC}(\mathrm{r}=-0.376, \mathrm{p}<0.001)$ at baseline. Moreover, changes in serum
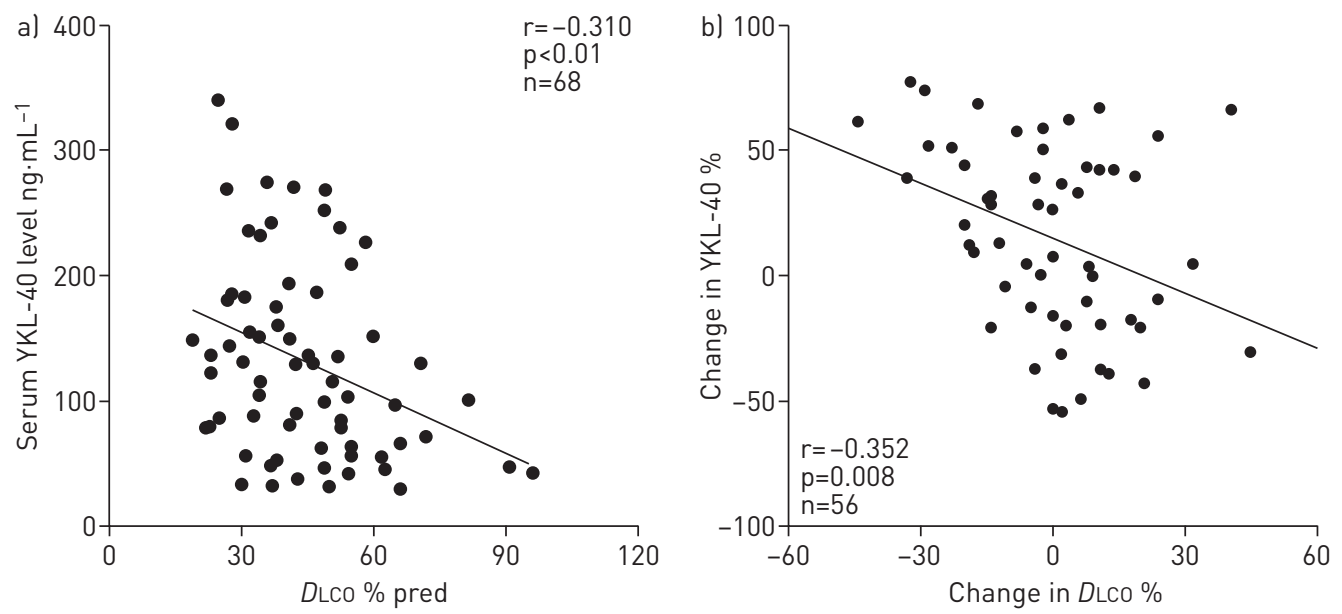

FIGURE 2 a) Correlation between baseline YKL-40 serum levels and diffusing capacity of the lung for carbon monoxide ( $D \mathrm{LCO})(\%$ pred) at baseline. Dots represent single patients. b) Correlation between change in serum YKL-40 and DLCO over time in 39 hypersensitivity pneumonitis patients. Dots represent 56 measurements performed in 39 patients at various time intervals ranging from 6 to 36 months. 
YKL-40 levels inversely correlated with changes in DLCO over time $(r=-0.352, p<0.001)$ (figure $2 b)$, but not with changes in FVC $(\mathrm{r}=0.221, \mathrm{p}=0.176)$.

\section{Serum YKL-40 levels and disease outcome in HP}

HP patients who experienced disease progression had higher baseline serum YKL-40 levels than those who remained stable during follow-up $\left(167 \pm 12\right.$ versus $\left.98 \pm 11 \mathrm{ng} \cdot \mathrm{mL}^{-1} ; \mathrm{p}<0.001\right)$ (figure $\left.3 \mathrm{a}\right)$. HP patients who died $(n=16)$ had higher baseline serum YKL-40 levels than those who survived $(n=56)(190 \pm 22$ versus $107 \pm 9 \mathrm{ng} \cdot \mathrm{mL}^{-1} ; \mathrm{p}<0.001$ ) (figure $3 \mathrm{~b}$ ).

Predictive value of baseline serum YKL-40 levels for disease progression in HP

Using ROC analysis, at a cut-off level of $>119 \mathrm{ng} \cdot \mathrm{mL}^{-1}$, serum YKL-40 levels showed the best sensitivity (81\%), specificity $(77 \%)$ and accuracy $(79 \%)$ to predict disease progression (AUC 0.797; $\mathrm{p}<0.001$ ). At a cut-off level of $>303 \mathrm{U} \cdot \mathrm{L}^{-1}$, serum LDH showed similar results (sensitivity $78 \%$, specificity $81 \%$, accuracy 79\%; AUC 0.811; $\mathrm{p}<0.001$ ) (figure 4a). The combination of the two cut-offs (serum YKL-40 $119 \mathrm{ng} \cdot \mathrm{mL}^{-1}$ and serum LDH $303 \mathrm{U} \cdot \mathrm{L}^{-1}$ ) yielded better results (sensitivity $81 \%$, specificity $91 \%$ and accuracy $80 \%$ ) to predict disease progression.

According to the best cut-off obtained by ROC analysis, we also divided all HP patients into a high YKL-40 level group $(\mathrm{n}=34)$, with baseline concentrations $>119 \mathrm{ng} \cdot \mathrm{mL}^{-1}$, and a low YKL-40 level group $(\mathrm{n}=38)$ (baseline concentrations $\left.\leqslant 119 \mathrm{ng} \cdot \mathrm{mL}^{-1}\right)$. The characteristics of patients stratified according to this cut-off are shown in table 2.

Univariate and multivariate analyses were performed to verify whether the identified cut-off predicts disease progression. In the univariate analysis, baseline serum YKL-40 levels $>119 \mathrm{ng} \cdot \mathrm{mL}^{-1}$ were associated with an increased risk of disease progression (hazard ratio (HR) 6.567; $\mathrm{p}<0.001$ ). In the multivariate analysis, serum YKL-40 levels $>119 \mathrm{ng} \cdot \mathrm{mL}^{-1}$ were independently associated with risk of disease progression after adjustment for age, sex, body mass index (BMI), smoking history, steroid therapy, disease type, baseline FVC, DLCO and serum LDH as covariates (HR 5.208; p=0.004) (table 3).

Kaplan-Meier analysis confirmed the predictive value of serum YKL-40 for disease progression in HP (figure 5a). At 5 years, the rate of disease progression of all HP patients was $40 \%$. The patients in the high YKL-40 group had a higher rate of disease progression (71\%) than those in the low YKL-40 group (13\%) (log-rank test, $\mathrm{p}<0.001)$.

\section{Predictive value of baseline serum YKL-40 levels for mortality in HP}

Using ROC analysis, at a cut-off level of $>150 \mathrm{ng} \cdot \mathrm{mL}^{-1}$, serum YKL-40 levels showed the best sensitivity (78\%), specificity (84\%) and accuracy (83\%) to predict death (AUC 0.787; p<0.001). For serum LDH, no predictive value for mortality was found $(\mathrm{p}>0.05)$ (figure $4 \mathrm{~b}$ ).

In the univariate analysis, baseline serum YKL-40 levels $>150 \mathrm{ng} \cdot \mathrm{mL}^{-1}$ were associated with an increased risk of death (HR 9.989; $\mathrm{p}<0.001$ ). In the multivariate analysis, serum YKL-40 levels $>150 \mathrm{ng} \cdot \mathrm{mL}^{-1}$ were independently associated with the risk of death after adjustment for age, sex, BMI, smoking history, steroid therapy, disease type, baseline FVC, DLCO and serum LDH as covariates (HR 6.413; p=0.013) (table 4).
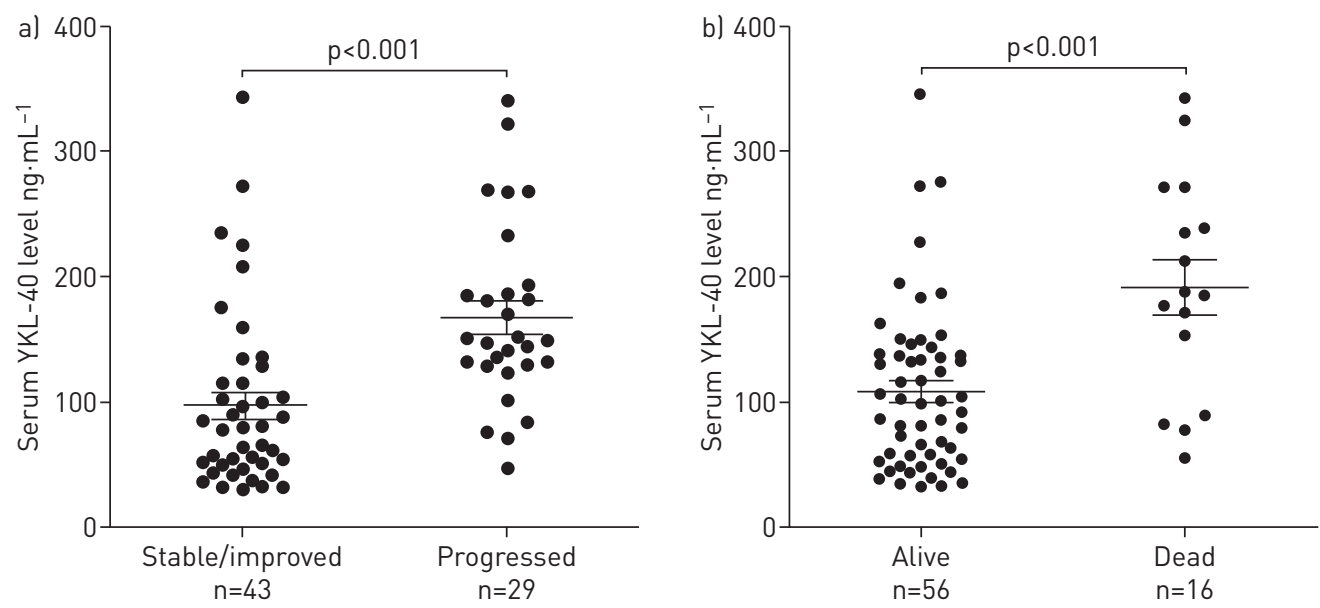

FIGURE 3 Comparisons of baseline serum YKL-40 levels in 72 hypersensitivity pneumonitis patients according to a) disease progression and b) survival. Dots represent single patients. Bars represent mean \pm SEM values. 

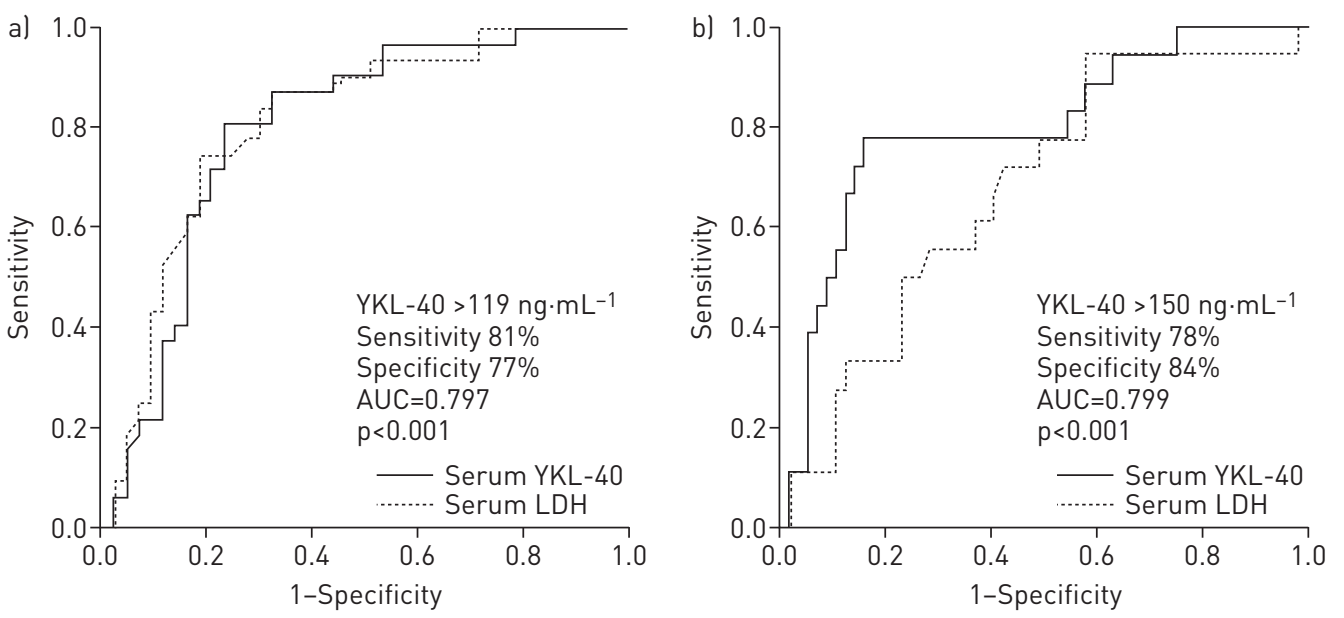

FIGURE 4 Receiver operating characteristic analysis showing the predictive value of baseline YKL-40 and lactate dehydrogenase (LDH) serum levels to predict al disease progression and b) mortality in 72 hypersensitivity pneumonitis patients. AUC: area under the curve.

TABLE 2 Characteristics of 72 hypersensitivity pneumonitis (HP) patients stratified according to serum YKL-40 predictive cut-off level for disease progression

YKL-40 $\leqslant 119 \mathrm{ng} \cdot \mathrm{mL}^{-1}$

$\mathrm{YKL}-40>119 \mathrm{ng} \cdot \mathrm{mL}^{-1}$

p-value

\begin{tabular}{|c|c|c|c|}
\hline Subjects & 38 & 34 & \\
\hline \multicolumn{4}{|l|}{ Demographics } \\
\hline Age years & $54 \pm 2$ & $61 \pm 2$ & NS \\
\hline Sex male/female & $15 / 23$ & $13 / 21$ & $N S^{\#}$ \\
\hline Smoking habits current/ex/nonsmoker & $0 / 12 / 26$ & $0 / 12 / 22$ & $N S^{\#}$ \\
\hline Steroid therapy yes/no & $19 / 19$ & $12 / 22$ & $N S^{\#}$ \\
\hline Disease type acute/subacute or chronic & $2 / 36$ & $9 / 25$ & $0.020^{\#}$ \\
\hline \multicolumn{4}{|l|}{ Disease outcome } \\
\hline Disease progression yes/no & $5 / 33$ & $24 / 10$ & $<0.001^{\#}$ \\
\hline HP-related death yes/no & $4 / 34$ & $12 / 22$ & $0.012^{\#}$ \\
\hline \multicolumn{4}{|l|}{ BALF cell differential } \\
\hline Total cells $\times 10^{4} \mathrm{~mL}^{-1}$ & $19 \pm 2$ & $32 \pm 2$ & 0.001 \\
\hline Macrophages $\times 10^{4} \mathrm{~mL}^{-1}$ & $9 \pm 2$ & $13 \pm 2$ & NS \\
\hline Lymphocytes $\times 10^{4} \mathrm{~mL}^{-1}$ & $8 \pm 1$ & $16 \pm 1$ & $<0.0001$ \\
\hline Granulocytes $\times 10^{4} \mathrm{~mL}^{-1}$ & $2 \pm 1$ & $4 \pm 1$ & NS \\
\hline Neutrophils $\times 10^{4} \mathrm{~mL}^{-1}$ & $0.4 \pm 0.2$ & $1.2 \pm 0.4$ & NS \\
\hline Eosinophils $\times 10^{4} \mathrm{~mL}^{-1}$ & $0.1 \pm 0.04$ & $0.3 \pm 0.08$ & NS \\
\hline Mast cells $\times 10^{4} \mathrm{~mL}^{-1}$ & $0.03 \pm 0.01$ & $0.1 \pm 0.06$ & NS \\
\hline \multicolumn{4}{|l|}{ Pulmonary function } \\
\hline FVC \% pred & $66 \pm 3$ & $57 \pm 3$ & 0.040 \\
\hline FEV $1 \%$ pred & $63 \pm 3$ & $59 \pm 3$ & NS \\
\hline DLCo \% pred & $45 \pm 2$ & $37 \pm 2$ & 0.013 \\
\hline TLC \% pred & $69 \pm 3$ & $70.5 \pm 3$ & NS \\
\hline \multicolumn{4}{|l|}{ Blood gas analysis } \\
\hline $\mathrm{PaO}_{2} \mathrm{mmHg}$ & $70 \pm 2$ & $68 \pm 2$ & NS \\
\hline $\mathrm{PA}-\mathrm{aO}_{2} \mathrm{mmHg}$ & $33 \pm 2$ & $35 \pm 2$ & NS \\
\hline $\mathrm{SaO}_{2} \%$ & $95 \pm 0.4$ & $94 \pm 0.5$ & NS \\
\hline \multicolumn{4}{|l|}{ Biomarkers } \\
\hline Serum YKL-40 $\mathrm{ng} \cdot \mathrm{mL}^{-1}$ & $68 \pm 4$ & $192 \pm 11$ & $<0.0001$ \\
\hline BALF YKL-40 $\mathrm{ng} \cdot \mathrm{mL}^{-1}$ & $14 \pm 5$ & $26 \pm 8$ & NS \\
\hline Serum LDH U. $\mathrm{L}^{-1}$ & $252 \pm 10$ & $328 \pm 11$ & $<0.0001$ \\
\hline
\end{tabular}

Data are presented as mean \pm SEM unless otherwise stated. NS: nonsignificant; BALF: bronchoalveolar lavage fluid; FVC: forced vital capacity; FEV1: forced expiratory volume in $1 \mathrm{~s}$; DLCO: diffusing capacity of the

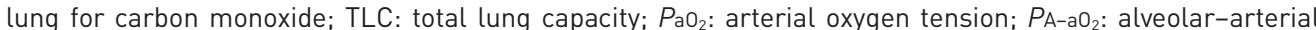
oxygen tension difference; $\mathrm{SaO}_{2}$ : arterial oxygen saturation; $\mathrm{LDH}$ : lactate dehydrogenase. ${ }^{\text {\# }}$ : Chi-square test or Fisher's exact test; for all other comparisons a t-test was used. 
TABLE 3 Univariate and multivariate Cox proportional analysis for the risk of disease progression in 72 hypersensitivity pneumonitis patients

\begin{tabular}{|c|c|c|c|}
\hline & Hazard ratio & $95 \% \mathrm{Cl}$ & p-value \\
\hline \multicolumn{4}{|l|}{ Univariate analysis } \\
\hline Serum YKL-40 ng $\cdot \mathrm{mL}^{-1}$ (continuous) & 1.009 & $1.005-1.014$ & $<0.001$ \\
\hline Serum YKL-40 >119 $\mathrm{ng} \cdot \mathrm{mL}^{-1}$ (binary) & 6.567 & $2.487-17.340$ & $<0.001$ \\
\hline Age years (continuous) & 1.028 & $1.000-1.058$ & 0.053 \\
\hline Sex $($ female $=1)$ & 0.991 & $0.466-2.106$ & NS \\
\hline Steroid therapy (positive $=1$ ) & 0.679 & $0.308-1.494$ & NS \\
\hline Smoking history (positive=1) & 0.997 & $0.974-1.021$ & NS \\
\hline Disease type (acute/subacute $=1$ ) & 0.328 & $0.136-0.788$ & 0.013 \\
\hline Total cells $\times 10^{4} \mathrm{~mL}^{-1}$ (continuous) & 1.026 & $0.998-1.055$ & NS \\
\hline Macrophages $\times 10^{4} \mathrm{~mL}^{-1}$ (continuous) & 1.013 & $0.981-1.047$ & NS \\
\hline Lymphocytes $\times 10^{4} \mathrm{~mL}^{-1}$ (continuous) & 1.079 & $1.015-1.148$ & 0.014 \\
\hline Granulocytes $\times 10^{4} \mathrm{~mL}^{-1}$ (continuous) & 1.099 & $0.941-1.284$ & NS \\
\hline Neutrophils $\times 10^{4} \mathrm{~mL}^{-1}$ (continuous) & 1.271 & $1.036-1.560$ & 0.022 \\
\hline Eosinophils $\times 10^{4} \mathrm{~mL}^{-1}$ (continuous) & 1.771 & $0.882-3.559$ & NS \\
\hline Mast cells $\times 10^{4} \mathrm{~mL}^{-1}$ (continuous) & 6.161 & $1.510-25.141$ & 0.011 \\
\hline FVC \% pred (continuous) & 0.989 & $0.963-1.015$ & NS \\
\hline FEV $1 \%$ pred (continuous) & 0.997 & $0.947-1.021$ & NS \\
\hline TLC \% pred (continuous) & 0.996 & $0.973-1.021$ & NS \\
\hline DLco \% pred (continuous) & 0.948 & $0.918-0.978$ & 0.001 \\
\hline $\mathrm{PaO}_{2} \mathrm{mmHg}$ (continuous) & 1.003 & $0.974-1.034$ & NS \\
\hline $\mathrm{PA}-\mathrm{aO}_{2} \mathrm{mmHg}$ (continuous) & 0.996 & $0.954-1.040$ & NS \\
\hline $\mathrm{SaO}_{2} \%$ (continuous) & 0.977 & $0.855-1.118$ & NS \\
\hline Serum LDH U.L ${ }^{-1}$ (continuous) & 1.010 & $1.005-1.015$ & $<0.001$ \\
\hline Serum LDH >303 U. $\mathrm{L}^{-1}$ (binary) & 4.715 & $2.085-10.665$ & $<0.001$ \\
\hline BALF YKL-40 $\mathrm{ng} \cdot \mathrm{mL}^{-1}$ (continuous) & 1.011 & $0.999-1.024$ & NS \\
\hline \multicolumn{4}{|l|}{ Multivariate analysis ${ }^{\#}$} \\
\hline Serum YKL-40 ng $\mathrm{mL}^{-1}$ (continuous) & 1.008 & $1.003-1.013$ & 0.002 \\
\hline D Lco \% pred (continuous) & 0.960 & $0.928-0.992$ & 0.016 \\
\hline Serum YKL-40 >119 $\mathrm{ng} \cdot \mathrm{mL}^{-1}$ (binary) & 5.208 & $1.713-15.835$ & 0.004 \\
\hline Serum LDH U.L $\mathrm{L}^{-1}$ (continuous) & 1.008 & $1.001-1.014$ & 0.017 \\
\hline
\end{tabular}

Data are presented as hazard ratios, representing the relative risk of developing disease progression as a specific characteristic at baseline. Backward and forward stepwise (conditional likelihood ratio) analyses were performed. NS: nonsignificant; FVC: forced vital capacity; FEV1: forced expiratory volume in $1 \mathrm{~s}$; TLC: total lung capacity; $D\left\llcorner C 0\right.$ : diffusing capacity of the lung for carbon monoxide; $\mathrm{PaO}_{2}$ : arterial oxygen tension: $\mathrm{PA}-\mathrm{aO}_{2}$ : alveolar-arterial oxygen tension difference; $\mathrm{SaO}_{2}$ : arterial oxygen saturation; $\mathrm{LDH}$ : lactate dehydrogenase; BALF: bronchoalveolar lavage fluid. \#: after adjustment for age, sex, body mass index, smoking history, steroid therapy, disease type, baseline FVC, DLCO and serum LDH.
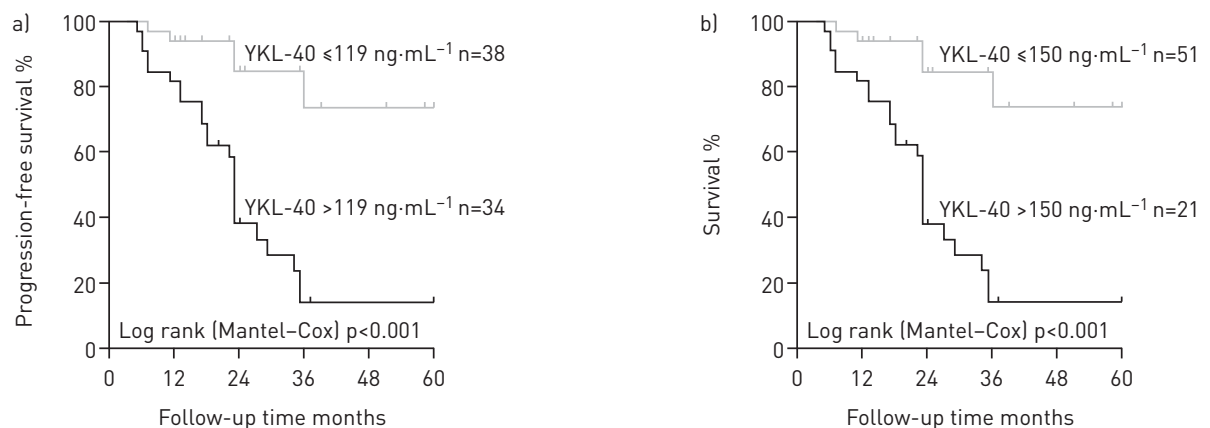

Number at risk

YKL-40 $\leqslant 119 \mathrm{ng} \cdot \mathrm{mL}^{-1} \quad 38 \quad 36 \quad 34 \quad 33 \quad 33 \quad 33$

$\mathrm{YKL}-40>119 \mathrm{ng} \cdot \mathrm{mL}^{-1} \quad 34 \quad 28 \quad 17 \quad 10 \quad 10 \quad 10$
Number at risk

$\begin{array}{lllllll}\mathrm{YKL}-40 \leqslant 119 \mathrm{ng} \cdot \mathrm{mL}^{-1} & 51 & 51 & 50 & 48 & 48 & 47 \\ \mathrm{YKL}-40>119 \mathrm{ng} \cdot \mathrm{mL}^{-1} & 21 & 17 & 15 & 12 & 12 & 9\end{array}$

FIGURE 5 Kaplan-Meier analysis showing al disease progression and b) survival rate according to serum YKL-40 levels in 72 hypersensitivity pneumonitis patients. The respective cut-off levels are indicated. 


\begin{tabular}{|c|c|c|c|}
\hline & Hazard ratio & $95 \% \mathrm{Cl}$ & p-value \\
\hline \multicolumn{4}{|l|}{ Univariate analysis } \\
\hline Serum YKL-40 ng $\cdot \mathrm{mL}^{-1}$ (continuous) & 1.010 & $1.005-1.015$ & $<0.001$ \\
\hline Serum YKL-40 >150 $\mathrm{ng} \cdot \mathrm{mL}^{-1}$ (binary) & 9.989 & $3.187-31.312$ & $<0.001$ \\
\hline Age years (continuous) & 1.107 & $1.047-1.171$ & $<0.001$ \\
\hline Sex (female $=1)$ & 1.068 & $0.387-2.947$ & NS \\
\hline Steroid therapy (positive $=1$ ) & 1.499 & $0.561-4.007$ & NS \\
\hline Smoking history ( positive $=1$ ) & 1.460 & $0.471-4.531$ & NS \\
\hline Disease type (acute/subacute $=1$ ) & 0.296 & $0.103-0.855$ & 0.025 \\
\hline Total cells $\times 10^{4} \mathrm{~mL}^{-1}$ (continuous) & 1.083 & $1.033-1.135$ & 0.001 \\
\hline Macrophages $\times 10^{4} \mathrm{~mL}^{-1}$ (continuous) & 1.069 & $1.024-1.117$ & 0.003 \\
\hline Lymphocytes $\times 10^{4} \cdot \mathrm{mL}^{-1}$ (continuous) & 1.044 & $0.958-1.317$ & NS \\
\hline Granulocytes $\times 10^{4} \cdot \mathrm{mL}^{-1}$ (continuous) & 1.087 & $0.933-1.266$ & NS \\
\hline Neutrophils $\times 10^{4} \mathrm{~mL}^{-1}$ (continuous) & 1.399 & $1.107-1.768$ & 0.005 \\
\hline Eosinophils $\times 10^{4} \mathrm{~mL}^{-1}$ (continuous) & 1.899 & $0.528-6.822$ & NS \\
\hline Mast cells $\times 10^{4} \mathrm{~mL}^{-1}$ (continuous) & 5.540 & $1.286-23.860$ & 0.022 \\
\hline FVC $\%$ pred (continuous) & 0.949 & $0.907-0.992$ & 0.021 \\
\hline FEV1, \% pred (continuous) & 0.975 & $0.941-1.011$ & NS \\
\hline TLC \% pred (continuous) & 0.989 & $0.955-1.024$ & NS \\
\hline DLco, \% pred (continuous) & 0.961 & $0.922-1.003$ & 0.001 \\
\hline $\mathrm{Pa02} \mathrm{mmHg}$ (continuous) & 1.011 & $0.969-1.054$ & NS \\
\hline $\mathrm{PA}-\mathrm{aO}_{2} \mathrm{mmHg}$ (continuous) & 0.989 & $0.945-1.036$ & NS \\
\hline $\mathrm{SaO}_{2} \%$ (continuous) & 1.013 & $0.839-1.223$ & NS \\
\hline Serum LDH U.L ${ }^{-1}$ (continuous) & 1.006 & $1.000-1.013$ & 0.058 \\
\hline BALF YKL-40 ng $\cdot \mathrm{mL}^{-1}$ (continuous) & 1.002 & $0.980-1.024$ & NS \\
\hline \multicolumn{4}{|l|}{ Multivariate analysis" } \\
\hline Serum YKL-40 ng $\cdot \mathrm{mL}^{-1}$ (continuous) & 1.010 & $1.001-1.019$ & 0.024 \\
\hline Age years (continuous) & 1.132 & $1.054-1.215$ & 0.001 \\
\hline FVC \% pred (continuous) & 0.945 & $0.901-0.990$ & 0.018 \\
\hline Serum YKL-40 >150 $\mathrm{ng} \cdot \mathrm{mL}^{-1}$ (binary) & 6.413 & $1.472-27.933$ & 0.013 \\
\hline Age years (continuous) & 1.137 & $1.055-1.226$ & 0.001 \\
\hline FVC \% pred (continuous) & 0.950 & $0.907-0.995$ & 0.029 \\
\hline
\end{tabular}

Data are presented as hazard ratios, representing the relative risk of death as a specific characteristic at baseline. Backward and forward stepwise (conditional LR) analyses were performed. NS: nonsignificant; FVC: forced vital capacity; FEV1: forced expiratory volume in $1 \mathrm{~s}$; TLC: total lung capacity; DLCo: diffusing capacity of the lung for carbon monoxide; $\mathrm{PaO}_{2}$ : arterial oxygen tension; $\mathrm{PA}_{\mathrm{A}-\mathrm{aO}}$ : alveolar-arterial oxygen tension difference; $\mathrm{SaO}_{2}$ : arterial oxygen saturation; $\mathrm{LDH}$ : lactate dehydrogenase; BALF: bronchoalveolar lavage fluid. "\#: after adjustment for age, sex, body mass index, smoking history, steroid therapy, disease type, baseline FVC, DLCO and serum LDH.

Kaplan-Meier analysis showed that higher serum YKL-40 was associated with mortality in HP (figure 5b). The mortality rate of all HP patients after 5 years was $22 \%$. The patients in the high YKL-40 group had a higher mortality rate (57\%) than those in the low YKL-40 group (8\%) (log-rank test, $\mathrm{p}<0.001)$.

\section{Discussion}

In this study, we show that serum and BALF YKL-40 levels are elevated in HP patients and other ILD patients compared with healthy controls, and correlate with pulmonary function tests (PFTs) and BALF cell counts. Moreover, serum YKL-40 was found to be an independent predictor of disease progression and mortality in HP. To the best of our knowledge, this is the first investigation of YKL-40 levels in serum and BALF and its potential role as a biomarker in HP.

HP is caused by repeated inhalation of organic particles; thus, antigen presentation and hypersensitivity reactions are involved in the development of this disorder [1, 3]. As previously reported, antigen-presenting cells produce YKL-40 in response to specific stimulation $[25,26]$. Thus, it can be speculated that activated antigen-presenting cells in the lung of HP patients are responsible for the elevated levels of YKL-40 in BALF of these patients. In support of this hypothesis, we found a positive correlation between serum YKL-40 levels and BALF lymphocyte counts in HP patients. Although lymphocytes cannot directly produce YKL-40, they can induce the production of interleukin-13, which can 
stimulate YKL-40 production in macrophages, dendritic cells and epithelial cells [7]. On the other hand, YKL-40 levels were higher in serum than BALF of patients with HP and other ILDs, suggesting a possible spill-over of YKL-40 from the bloodstream to the alveolar space. This has also been reported in a previous study on YKL-40 in IPF [23]. As postulated for asthma [27], the elevated concentration of YKL-40 in serum of HP patients may reflect the activity of antigen-presenting cells and the systemic immune response. In line with this hypothesis, serum and BALF levels of YKL-40 were significantly higher in acute/subacute HP patients than in those with chronic HP.

Serum YKL-40 was inversely correlated with PFTs at baseline; this finding has also been observed in IPF [13] and sarcoidosis [28]. Interestingly, change in YKL-40 levels in serum also correlated with change in DLCO over time. Moreover, the serum level of YKL-40 correlated well with LDH, a nonspecific marker of tissue injury widely used to monitor the course of acute lung disease and chronic fibrotic disease [29].

We found that HP patients with progressive disease had higher levels of YKL-40 than those who remained stable or improved. Serum YKL-40 levels $>119 \mathrm{ng} \cdot \mathrm{mL}^{-1}$ predicted disease progression with a sensitivity of $81 \%$ and specificity of $77 \%$, similar to serum LDH (cut-off $303 \mathrm{U} \cdot \mathrm{L}^{-1}$, sensitivity $78 \%$, specificity $81 \%$ ). Moreover, the combined assessment of serum YKL-40 and LDH provided better results (sensitivity $81 \%$, specificity 91\%) than either single marker alone. By using the identified cut-off to stratify the patients, those with serum YKL-40 levels $>119 \mathrm{ng} \cdot \mathrm{mL}^{-1}$ had worse PFTs and a higher rate of disease progression (71\%) than those with serum YKL-40 $\leqslant 119 \mathrm{ng} \cdot \mathrm{mL}^{-1}(13 \%)$. The multivariate analysis confirmed that both a serum YKL-40 level $>119 \mathrm{ng} \cdot \mathrm{mL}^{-1}$ and YKL-40 as a continuous variable were independently associated with disease progression, even after adjusting for covariates. The same was not observed for serum LDH.

Moreover, HP patients with serum YKL-40 levels $>150 \mathrm{ng} \cdot \mathrm{mL}^{-1}$ had worse PFTs and a worse survival (57\% mortality rate) compared with those with $\leqslant 150 \mathrm{ng} \cdot \mathrm{mL}^{-1}$ ( $8 \%$ mortality rate). The multivariate analysis confirmed that a serum YKL-40 level $>150 \mathrm{ng} \cdot \mathrm{mL}^{-1}$ was associated with mortality, even after adjusting for covariates. This predictive cut-off for survival in our cohort is approximately two-fold higher than that reported in IPF patients $\left(79 \mathrm{ng} \cdot \mathrm{mL}^{-1}\right)$ [23]. This suggests that production of YKL-40 level is enhanced in HP patients with active disease, and correlates with outcome.

The thresholds for serum YKL-40 described in this study might be different in other cohorts. However, this does not impact our main finding that HP patients with high serum YKL-40 levels at baseline may have a higher rate of disease progression (or death) than those with lower levels. In recent years, there has been increasing interest in defining accurate predictors of outcome in HP: a higher fibrosis score on HRCT [30], increasing severity of traction bronchiectasis [31], lower lymphocyte levels in BALF [32] and a usual interstitial pneumonia-like pattern on histology $[31,32]$ have been found to correlate with disease outcome. Whereas MYAZAKI et al. [32] previously identified a low lymphocyte level in BALF as a potential risk factor for acute exacerbation of HP, in our cohort a higher BALF lymphocyte count was seen in the HP group with high YKL-40 levels, driven by the higher number of patients with acute/subacute disease in this group. However, univariate and multivariate analyses demonstrated that lymphocyte count was not an independent predictive factor for disease progression or survival.

Besides clinical characteristics, two biomarkers have been studied in the past to evaluate outcome in HP patients: KL-6 [33, 34] and CCL17 [35]. Serum KL-6 has been shown to predict disease progression and survival in HP patients $[33,34]$, whereas serum CCL17 was shown to be a predictor of disease progression but not of survival [35].

With regard to the factors responsible for YKL-40 variability, we found, in agreement with others [36, 37], that YKL-40 serum levels correlated with age in our entire cohort, but the correlation was weak (data not shown). As reported previously [36,38], we also found that former and current smokers had higher serum YKL-40 levels than never-smokers. Cigarette smoke increases the permeability of the air-blood membrane [39] and could increase both production and spill-over of YKL-40; it has been shown that YKL-40 expression is increased in alveolar macrophages and epithelial cells in lung tissue of cigarette smokers with chronic obstructive pulmonary disease and cigarette smoke-exposed mice [37]. We believe that smoking habits should be taken into consideration when interpreting YKL-40 serum levels. The relevance of this aspect for HP patients is low, since the majority of HP patients are nonsmokers. Moreover, we observed that corticosteroid-treated patients had significantly lower levels of serum YKL-40 levels than untreated patients, suggesting that corticosteroids may suppress YKL-40 release [40, 41]. This observation has not been reported in other studies of ILD patients and could be of clinical utility in assessing the clinical response to steroid treatment in HP patients.

Despite the novel findings of this study, it has several limitations. First, the healthy controls were younger than the ILD patients, and this difference could affect the respective serum levels of YKL-40. Secondly, only limited data on serum YKL-40 levels during follow-up were available, and these data did not allow us 
to assess the clinical value of serial YKL-40 measurements. Thirdly, baseline single nucleotide polymorphisms in the chitinase 3-like 1 (CHI3L1) encoding gene, known to influence YKL-40 levels in serum, were not determined [23]. Finally, immunohistochemistry to demonstrate YKL-40 in lung tissue obtained by biopsy was not performed, and therefore we can only speculate on the source and localisation of this protein in HP and other ILDs.

In conclusion, this study indicates that serum YKL-40 can be a prognostic biomarker in HP, suggesting a clinical role in predicting disease progression and survival, but its serum levels should be carefully interpreted in light of the confounding factors. Although the data are promising, a multicentre validation study is necessary to determine whether serum YKL-40 measurements should be routinely used in HP.

\section{Acknowledgements}

Authors' contributions: X. Long and F. Bonella contributed to the conception and design of the study, sample collection, measurement of biomarkers, analysis and interpretation of the data, and drafting and finalisation of the manuscript. $\mathrm{X}$. He collected samples and performed biomarker measurement. S. Ohshimo and M. Griese contributed to analysis and interpretation of the data. R. Sarria and J. Guzman contributed to the conception and design of the study, and drafting of the manuscript. U. Costabel contributed to the conception and design of the study, and finalisation of the manuscript. All authors have read and approved the final manuscript.

\section{References}

1 Costabel U, Bonella F, Guzman J. Chronic hypersensitivity pneumonitis. Clin Chest Med 2012; 33: 151-163.

2 Selman M. Hypersensitivity pneumonitis: a multifaceted deceiving disorder. Clin Chest Med 2004; 25 : $531-547$.

3 Mohr LC. Hypersensitivity pneumonitis. Curr Opin Pulm Med 2004; 10: 401-411.

4 Jinta T, Miyazaki Y, Kishi M, et al. The pathogenesis of chronic hypersensitivity pneumonitis in common with idiopathic pulmonary fibrosis: expression of apoptotic markers. Am J Clin Pathol 2010; 134: 613-620.

5 Hakala BE, White C, Recklies AD. Human cartilage gp-39, a major secretory product of articular chondrocytes and synovial cells, is a mammalian member of a chitinase protein family. J Biol Chem 1993; 268: 25803-25810.

6 Lee CG, Cruz CSD, Herzog E, et al. YKL-40, a chitinase-like protein at the intersection of inflammation and remodeling. Am J Respir Crit Care Med 2012; 185: 692-694.

7 Lee CG, Da Silva CA, Dela Cruz CS, et al. Role of chitin and chitinase/chitinase-like proteins in inflammation, tissue remodeling, and injury. Annu Rev Physiol 2011; 73: 479-501.

8 Korthagen NM, van Moorsel CHM, Zanen P, et al. Evaluation of circulating YKL-40 levels in idiopathic interstitial pneumonias. Lung 2014; 192: 975-980.

9 Berres ML, Papen S, Pauels K, et al. A functional variation in CHI3L1 is associated with severity of liver fibrosis and YKL-40 serum levels in chronic hepatitis C infection. J Hepatol 2009; 50: 370-376.

10 Bara I, Ozier A, Girodet PO, et al. Role of YKL-40 in bronchial smooth muscle remodeling in asthma. Am J Respir Crit Care Med 2012; 185: 715-722.

11 Erzin Y, Uzun H, Karatas A, et al. Serum YKL-40 as a marker of disease activity and stricture formation in patients with Crohn's disease. J Gastroenterol Hepatol 2008; 23(8 Pt 2): e357-e362.

12 Kazakova M, Batalov A, Deneva T, et al. Relationship between sonographic parameters and YKL-40 levels in rheumatoid arthritis. Rheumatol Int 2013; 33: 341-346.

13 Furuhashi K, Suda T, Nakamura Y, et al. Increased expression of YKL-40, a chitinase-like protein, in serum and lung of patients with idiopathic pulmonary fibrosis. Respir Med 2010; 104: 1204-1210.

14 Johansen JS, Milman N, Hansen M, et al. Increased serum YKL-40 in patients with pulmonary sarcoidosis-a potential marker of disease activity? Respir Med 2005; 99: 396-402.

15 Long X, Costabel U, He X, et al. Serum YKL-40 as a candidate biomarker for hypersensitivity pneumonitis. Pneumologie 2015; 69: S5.

16 Lacasse Y, Selman M, Costabel U, et al. Clinical diagnosis of hypersensitivity pneumonitis. Am J Respir Crit Care Med 2003; 168: 952-958.

17 Raghu G, Collard HR, Egan JJ, et al. An Official ATS/ERS/JRS/ALAT Statement: Idiopathic Pulmonary Fibrosis: Evidence-Based Guidelines for Diagnosis and Management. Am J Respir Crit Care Med 2011; 183: 788-824.

18 ATS/ERS. American Thoracic Society/European Respiratory Society International Multidisciplinary Consensus Classification of the Idiopathic Interstitial Pneumonias. Am J Respir Crit Care Med 2002; 165: 277-304.

19 Travis WD, Costabel U, Hansell DM, et al. An Official American Thoracic Society/European Respiratory Society Statement: Update of the International Multidisciplinary Classification of the Idiopathic Interstitial Pneumonias. Am J Respir Crit Care Med 2013; 188: 733-748.

20 Bonella F, Ohshimo S, Miaotian C, et al. Serum KL-6 is a predictor of outcome in pulmonary alveolar proteinosis. Orphanet J Rare Dis 2013; 8: 53.

21 Schmidt SL, Tayob N, Han MK, et al. Predicting pulmonary fibrosis disease course from past trends in pulmonary function. Chest 2014; 145: 579-585.

22 Klech H, Pohl W. Technical recommendations and guidelines for bronchoalveolar lavage (BAL). Report of the European Society of Pneumology Task Group. Eur Respir J 1989; 2: 561-585.

23 Korthagen NM, van Moorsel CHM, Barlo NP, et al. Serum and BALF YKL-40 levels are predictors of survival in idiopathic pulmonary fibrosis. Respir Med 2011; 105: 106-113.

24 Standardized lung function testing. Official statement of the European Respiratory Society. Eur Respir J Suppl 1993; 16: 1-100.

25 Liu CY, Li Q, Zhou XD, et al. The chitinase-like protein YKL-40 increases mucin5AC production in human bronchial epithelial cells. Exp Cell Res 2013; 319: 2866-2873.

26 Riabov V, Gudima A, Wang N, et al. Role of tumor associated macrophages in tumor angiogenesis and lymphangiogenesis. Physiology 2014; 5: 75.

27 Tang H, Fang Z, Sun Y, et al. YKL-40 in asthmatic patients, and its correlations with exacerbation, eosinophils and immunoglobulin E. Eur Respir J 2010; 35: 757-760. 
28 Kruit A, Grutters JC, Ruven HJT, et al. A CHI3L1 gene polymorphism is associated with serum levels of YKL-40 a novel sarcoidosis marker. Respir Med 2007; 101: 1563-1571.

29 Drent M, Cobben NA, Henderson RF, et al. Usefulness of lactate dehydrogenase and its isoenzymes as indicators of lung damage or inflammation. Eur Respir J 1996; 9: 1736-1742.

30 Mooney JJ, Elicker BM, Urbania TH, et al. Radiographic fibrosis score predicts survival in hypersensitivity pneumonitis. Chest 2013; 144: 586-592.

31 Walsh SLF, Sverzellati N, Devaraj A, et al. Chronic hypersensitivity pneumonitis: high resolution computed tomography patterns and pulmonary function indices as prognostic determinants. Eur Radiol 2012; 22: 1672-1679.

32 Miyazaki Y, Tateishi T, Akashi T, et al. Clinical predictors and histologic appearance of acute exacerbations in chronic hypersensitivity pneumonitis. Chest 2008; 134: 1265-1270.

33 Ishikawa N, Hattori N, Yokoyama A, et al. Utility of KL-6/MUC1 in the clinical management of interstitial lung diseases. Respir Investig 2012; 50: 3-13.

34 Ohnishi H, Miyamoto S, Kawase S, et al. Seasonal variation of serum KL-6 concentrations is greater in patients with hypersensitivity pneumonitis. BMC Pulm Med 2014; 14: 129.

35 Miyazaki Y, Unoura K, Tateishi T, et al. Higher serum CCL17 may be a promising predictor of acute exacerbations in chronic hypersensitivity pneumonitis. Respir Res 2013; 14: 57.

36 Guerra S, Halonen M, Sherrill DL, et al. The relation of circulating YKL-40 to levels and decline of lung function in adult life. Respir Med 2013; 107: 1923-1930.

37 Matsuura H, Hartl D, Kang MJ, et al. Role of breast regression protein-39 in the pathogenesis of cigarette smoke-induced inflammation and emphysema. Am J Respir Cell Mol Biol 2011; 44: 777-786.

38 Sakazaki Y, Hoshino T, Takei S, et al. Overexpression of chitinase 3-like 1/YKL-40 in lung-specific IL-18-transgenic mice, smokers and COPD. PLoS One 2011; 6: e24177.

39 Li XY, Donaldson K, Rahman I, et al. An investigation of the role of glutathione in increased epithelial permeability induced by cigarette smoke in vivo and in vitro. Am J Respir Crit Care Med 1994; 149: 1518-1525.

40 Johansen JS, Stoltenberg M, Hansen M, et al. Serum YKL-40 concentrations in patients with rheumatoid arthritis: relation to disease activity. Rheumatology (Oxford) 1999; 38: 618-626.

41 Kzhyshkowska J, Mamidi S, Gratchev A, et al. Novel stabilin-1 interacting chitinase-like protein (SI-CLP) is up-regulated in alternatively activated macrophages and secreted via lysosomal pathway. Blood 2006; 107: 3221-3228. 